\title{
Con we improve the dose distribution for single or multi-lumen breast balloons used for Accelerated Partial Breast Irradiation?
}

\author{
Janusz Skowronek, MD, PhD ${ }^{12}$, Grzegorz Bielęda, MSc', Piotr Łaski, MD, PhD4, Witold Kycler, MD, PhD ${ }^{4}$ \\ IBrachytherapy Department, Greater Poland Cancer Center, 2Electroradiology Department, Poznan University of Medical Sciences, \\ 3 Medical Physics Department, Greater Poland Cancer Center, ${ }^{42}$ nd Surgery Department, Greater Poland Cancer Center, Poznan, Poland
}

\begin{abstract}
Purpose: The aim of the study was to verify dose distribution parameters for multi-lumen, and artificially created single-lumen balloon applicator used for the same patient with two optimization algorithms: inverse planning simulated annealing (IPSA) and dose point optimization with distance option.

Material and methods: Group of 24 patients with multi-lumen balloon applied were investigated. Each patient received 10 fractions of $3.4 \mathrm{~Gy}$ ( 2 fractions daily). For every patient, four treatment plans were prepared. Firstly, for fivelumen balloon optimized with IPSA algorithm and optimization parameters adjusted for each case. Secondly, for the same applicator optimized with dose point optimization and distant option. Two other plans were prepared for singlelumen applicator, created by removing four peripheral lumens, optimized with both algorithms.

Results: The highest $\mathrm{D}_{95}$ parameter was obtained for plans optimized with IPSA algorithm, mean value 99.3 percent of prescribed dose, and it was significantly higher than plans optimized with dose point algorithm $(\mathrm{mean}=83.50 \%$, $p<0.0001$ ), IPSA single-lumen balloon plan (mean $=83.50 \%, p=0.0037$ ) and optimized to dose point single-lumen balloon (mean $=85.51 \%, p<0.0001)$. There were no statistically significant differences concerning maximum doses distributed to skin surface for neither application nor optimization method. Volumes receiving $200 \%$ of prescribed dose in PTV were higher for multi-lumen balloon dose point optimized plans (mean $=8.78 \%$ ), than for other plans (IPSA multi-lumen balloon plan: mean $=7.37 \%, p<0.0001$, single-lumen IPSA: mean $=7.20 \%, p<0.0001$, single-lumen dose point: mean $=7.19 \%, p<0.0001)$.
\end{abstract}

Conclusions: Basing on performed survey, better dose distribution parameters are obtained for patients with multi-lumen balloon applied and optimized using IPSA algorithm with individualized optimization parameters.

Key words: APBI, brachytherapy, breast cancer, Contura, IPSA.

\section{Purpose}

Breast-conserving therapy (BCT) has traditionally included whole breast irradiation after tumor lumpectomy. This approach is based on the premise that irradiation will eliminate residual foci of tumor adjacent to the surgical bed, and will reduce the risk of recurrence from areas in situ or infiltrating carcinoma elsewhere in the breast. Growing body of data provides compelling evidence that irradiation after lumpectomy is effective only at the site of initial involvement, and thus may safely be limited to tissues immediately surrounding the excision cavity, with an expected local control rate comparable to the rate achieved with whole-breast irradiation [1-4]. Most studies of phases I and II using this technique have demonstrated acceptable 5- and 10-year rates of local control, and good cosmetic effect in highly selected, low-risk patients $[5,6]$. The MammoSite ${ }^{\circledR}$ applicator (Hologic Inc., Bed- ford, MA, USA) was one of the first devices designed to address these issues. One of the major limitations with the use of the MammoSite to deliver APBI, relates to its single lumen design, and the fixed relationship between the geometry of balloon placement and the dose delivered. The Contura ${ }^{\circledR}$ multi-lumen balloon (SenoRx, Inc., Aliso Viejo, CA, USA) was designed to provide additional options to achieve more idealized dosimetric goals (Fig. 1) [7]. Through the use of four additional lumens that are offset from the single central catheter lumen, dose shaping is possible. Published dosimetric data have demonstrated the device's ability to significantly reduce skin and rib doses, and improve on other treatment restrictions (as a result of insufficient skin spacing or suboptimal conformance [7-11]). Basing on our own studies, we verified dose distribution parameters for Contura ${ }^{\circledR}($ SenoRx, Inc.), and artificially created single-lumen balloon applicator used for the same patient, applying two optimization algo-
Address for correspondence: Janusz Skowronek, MD, PhD, Asst. Prof., Brachytherapy Department, Greater Poland Cancer Center, 15 Garbary Street, 61-866 Poznan, Poland, phone: +48 618850818, +48 602618538, fax: +48 618850834, « e-mail: janusz.skowronek@wco.pl
Received: 28.05 .2013

Accepted: 10.08 .2013

Published: 26.09 .2013 
rithms: Inverse Planning Simulated Annealing (IPSA) and dose point optimization with distance option.

\section{Material and methods}

From November 2009 to March 2011 we have treated 24 patients using Contura ${ }^{\circledR}$ multi-lumen breast balloon (SenoRx, Inc.). Patients were treated with total dose of 34 Gy in 10 fraction, 2 fractions per day in 5 consecutive days, excluding weekends in accelerated partial breast irradiation (APBI) mode. High-dose-rate (HDR) technique was used. Treatment plans were prepared using computed tomography (CT) imaging with slice thickness of $1 \mathrm{~mm}$. PTV_EVAL was the volume in which the dose was reported, and was limited to $5 \mathrm{~mm}$ from the skin. It was created by adding $10 \mathrm{~mm}$ margin around the balloon. If the skin or pectoral muscle were overlapping the margin creating planning target volume (PTV), the overlapping parts were subtracted (Fig. 2). The balloon was subtracted as well, as the doses inside the balloon were unlimited. Maximal doses in ribs and skin were calculated.

Before each fraction, the dressing covering the breast of the patient and the applicator was removed. The configuration of the orientation line on the applicator, in relation to the line drawn on skin of the patient was checked just before the CT planning. Furthermore, before the CT planning, the fluid around the balloon was vacuumed. Air and fluid was removed before each fraction to keep the condition from the time of planning.

Oncentra Masterplan vs 3.2 ${ }^{\circledR}$ (Nucletron, an Elekta company, Elekta AB, Stockholm, Sweden) treatment planning system was used for planning. Dose distribution for treatment was calculated using IPSA algorithm with individually adapted optimization parameters (ML IPSA). There were three additional virtual plans prepared for each patient in order to compare dose distribution, using single and multi-lumen devices used for APBI. The first virtual plan was calculated for the same device using dose points optimization with distance option (ML DP). Next plan for single lumen applicator was prepared by deleting the radial lumens from the applicator in treatment planning system. The first plan for virtual applicator was optimized using IPSA algorithm (SL IPSA). The last plan was prepared for

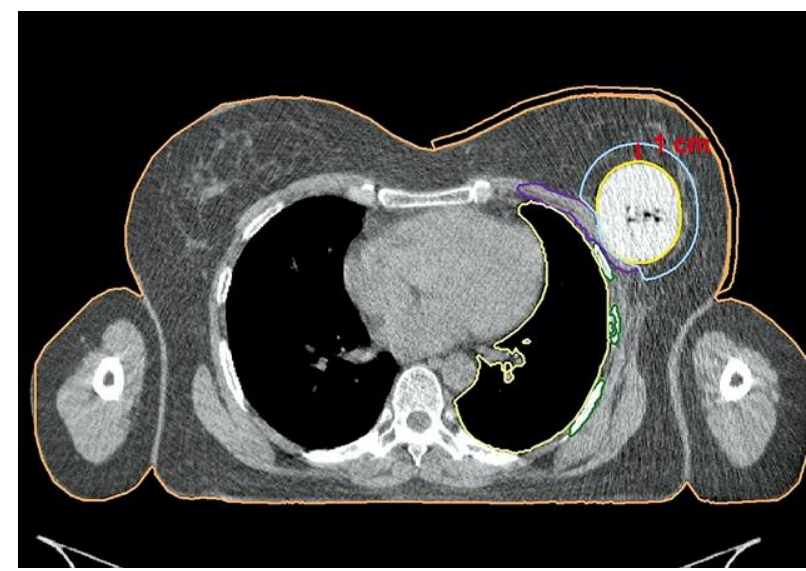

Fig. 2. Anatomy reconstruction in Contura ${ }^{\circledR}$ application

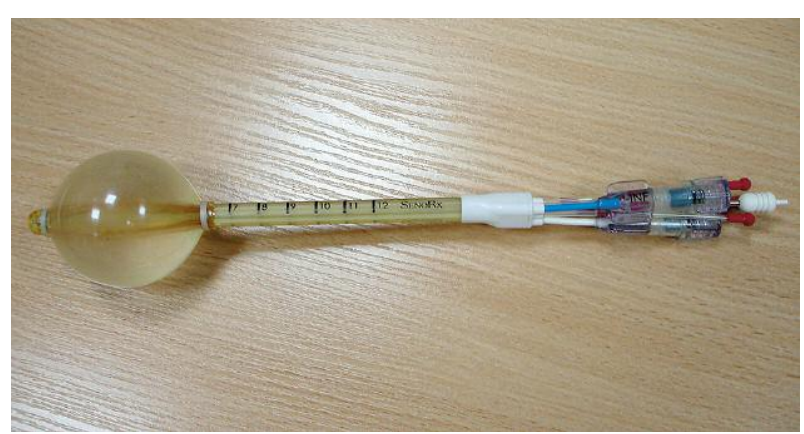

Fig. 1. Contura ${ }^{\circledR}$ multi-lumen balloon (SenoRx, Inc., Aliso Viejo, CA, USA)

single lumen applicator, and was optimized using dose points optimization (SL DP). To compare the dose optimization algorithms, no manual optimization was performed, as physicist interference and dose modification would compromise the results of TPS calculations. Real plans after acceptance were optimized only with IPSA algorithm without any other optimization. The same way, a plan using IPSA optimization for single lumen applicator was optimized. Only the IPSA parameters were modified. We compared the same way single and multi-lumen applicators. Our goal was to verify the implemented algorithms. Exemplary dose distribution is presented in Figure 3.

Primary dosimetric goals to accept treatment plan were defined as: (a) $\mathrm{D}_{95}$ in PTV_EVAL $>95 \%$; (b) $\mathrm{V}_{150}<50$ cc; (c) $\mathrm{V}_{200}<10$ cc; (d) Skin $\mathrm{D}_{\max }<125 \%$; (e) Rib $\mathrm{D}_{\max }<125 \%$. Plans were recognized as acceptable, if suboptimal application conditions prevented as from achieving primary goals, and the restrictions had to be lowered to: (a) $\mathrm{D}_{90}$ in PTV_EVAL $>90 \%$ prescribed dose; (b) Skin $D_{\max }$ $<145 \%$ prescribed dose, with the remaining parameters unchanged.

To verify these plans, dose distribution parameters representing doses in target volume and in organs at risk were compared. Dose distribution parameters were obtained from dose volume histograms. Within our interest were the following: $\mathrm{D}_{95}, \mathrm{D}_{90}, \mathrm{~V}_{150}, \mathrm{~V}_{200}, \mathrm{D}_{10}, \mathrm{D}_{50}$, and doses to OARs $-D_{\max }$ rib and $D_{\max }$ skin. All the parameters that represented the doses were taken into account as percent of prescribed dose, except of $\mathrm{V}_{150}$ and $\mathrm{V}_{200}$, which were presented in $\mathrm{cm}^{3}$.

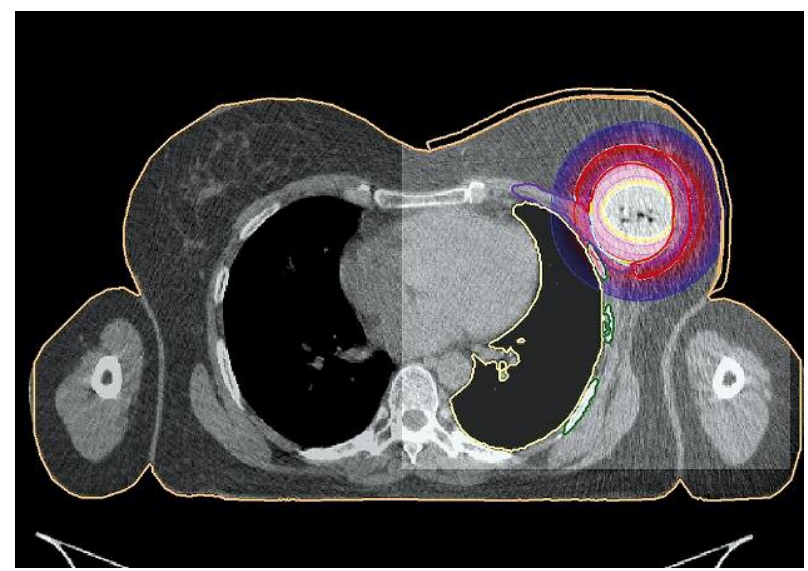

Fig. 3. Dose distribution visualization 
Table 1. Dose distribution parameters (mean values and SD) for real and virtual plans

\begin{tabular}{lcccc} 
& Multi-lumen IPSA & Multi-lumen DP & Single-lumen IPSA & Single-lumen DP \\
\hline $\mathrm{D}_{95}[\%]$ & $99.32 \pm 6.75$ & $83.50 \pm 8.16$ & $97.82 \pm 7.51$ & $85.52 \pm 10.21$ \\
\hline $\mathrm{D}_{90}[\%]$ & $99.95 \pm 19.61$ & $88.40 \pm 7.96$ & $102.56 \pm 7.13$ & $90.27 \pm 9.82$ \\
\hline $\mathrm{D}_{\max }$ skin [\%] & $85.83 \pm 28.59$ & $81.34 \pm 28.43$ & $85.31 \pm 32.31$ & $85.07 \pm 43.20$ \\
\hline $\mathrm{D}_{\max }$ rib [\%] & $92.52 \pm 33.86$ & $84.59 \pm 24.13$ & $105.56 \pm 33.34$ & $96.05 \pm 34.59$ \\
\hline $\mathrm{D}_{50}[\%]$ & $133.41 \pm 18.96$ & $118.70 \pm 21.06$ & $132.28 \pm 21.15$ & $119.99 \pm 23.86$ \\
\hline $\mathrm{D}_{10}[\%]$ & $190.62 \pm 9.63$ & $193.96 \pm 18.46$ & $190.07 \pm 12.42$ & $185.05 \pm 14.21$ \\
\hline $\mathrm{V}_{150}\left[\mathrm{~cm}^{3}\right]$ & $34.72 \pm 4.84$ & $24.20 \pm 4.01$ & $33.60 \pm 5.25$ & $24.45 \pm 4.76$ \\
\hline $\mathrm{V}_{200}\left[\mathrm{~cm}^{3}\right]$ & $7.37 \pm 4.25$ & $8.78 \pm 3.04$ & $7.20 \pm 4.72$ & $7.19 \pm 3.44$
\end{tabular}

IPSA - Inverse Planning Simulated Annealing, DP - dose points optimization, D - dose (dose that covers \% of the target volume), $D_{\text {max }}-$ maximal dose received by skin or rib, $V$-volume $\left(V_{150}\right.$, that is the percentage of the target volume that has received $50 \%$ more than the prescribed dose, $V_{200}-100 \%$ more)

$\mathrm{D}_{95}$

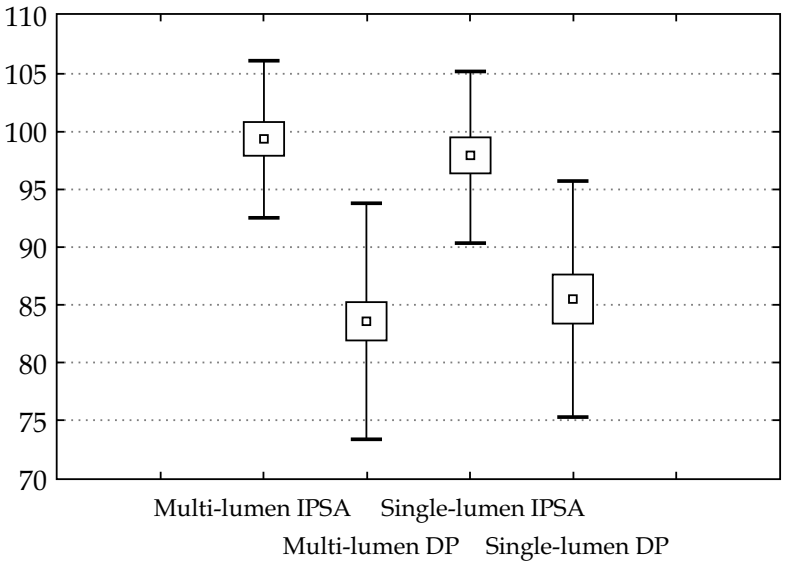

$\mathrm{D}_{\max }$ skin

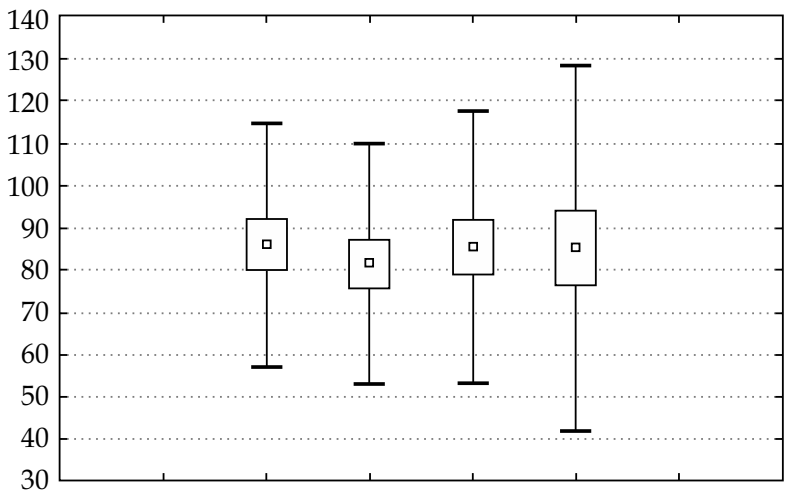

Multi-lumen IPSA Single-lumen IPSA

Multi-lumen DP Single-lumen DP
$\mathrm{D}_{50}$

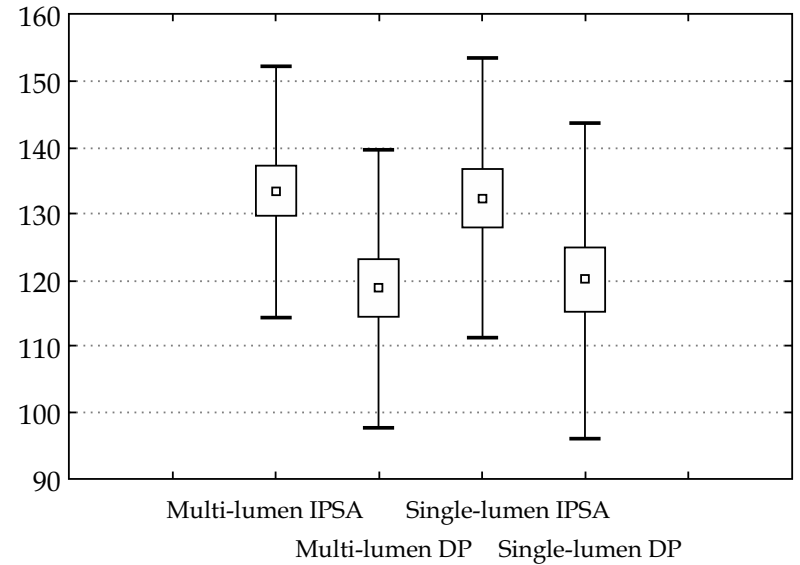

$\mathrm{V}_{150}$

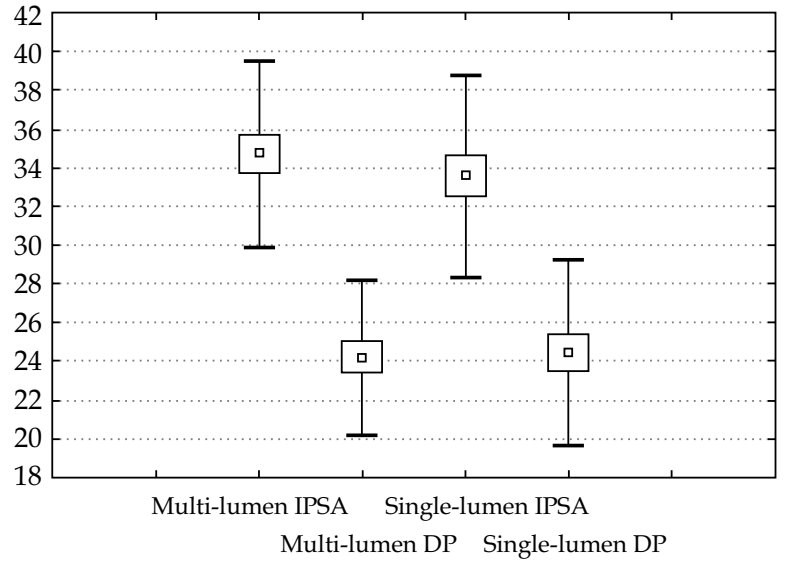

Fig. 4. Mean values and SD for doses deposited to target $\left(D_{95}, D_{50}, V_{150}\right)$ and OAR $\left(D_{\max }\right.$ skin) for plans prepared using different methods

The dose distribution parameters from three virtual plan sets, with the original plans prepared to treat the patients were compared using Student's $t$-test $(\alpha<0.05)$.

\section{Results}

A total of 24 patients were treated using multi-lumen intracavitary breast balloon. The mean values with the stan- dard deviations of achieved dose distribution parameters are presented in Table 1 and Figure 4 . Table 2 contains p value of Student's $t$-test for comparison of dose distribution obtained for realized plan versus values calculated for virtual plans.

The highest values of $\mathrm{D}_{95}$ was obtained for IPSA optimized plan for multi-lumen (ML IPSA) applicator (99.32 \pm $6.75 \%)$. This difference was statistically significant for all 
three virtual plans: multi-lumen dose point optimized (ML DP), single-lumen IPSA optimized (SL IPSA), singlelumen dose point optimized (SL DP), $p<0.0001, p=0.0367$ and $p<0.0001$, respectively. Only the values of maximal dose to skin were comparable for all the plans. No statistical significant differences were found (ML DP $p=0.0816$, SL IPSA $p=0.7131$, SL DP $p=0.8763$ ).

SL IPSA plans were not significantly different from ML IPSA plans, taking into account mean values of $\mathrm{D}_{90}(p=$ $0.544), \mathrm{D}_{50}(p=0.2245)$ and $\mathrm{V}_{150}(p=0.2369)$. The values of the other two virtual planning results, provided outcomes statistically different from obtained using ML IPSA for these DVH parameters $\mathrm{D}_{90}(p=0.0145, p=0.0350), \mathrm{D}_{50}(p<0.0001$, $p<0.0001)$, and $\mathrm{V}_{150}(p<0.0001, p<0.0001)$ for ML DP and SL DP, respectively.

\section{Discussion}

APBI using intracavitary breast balloons is a technique which gives as opportunity to treat patients in short period of time. The main advantage of this method is that intracavitary application and high dose gradient permit us to irradiate the target area with prescribed dose, while conserving the organs at risk. The cosmetic outcome of the treatment is also very important, so the lowest possible skin doses and calculation of the smallest high-dose volumes are crucial [12-17].

The multi-lumen construction of the applicator gives us broader possibility to adjust the dose distribution in the treated area [12,18-20]. Optimization of dose distribution using multi-lumen device had more degrees of freedom, as more active source dwell positions are available. The plans calculated with IPSA algorithm characterized with significantly better dose coverage of the treated area while keeping doses to skin surface at similar level.

In this analysis, compared with standard single-lumen, balloon-based brachytherapy, potential improvements in dosimetric capabilities (i.e., reduced skin dose, improved PTV_EVAL coverage, and normal tissue avoidance) were realized. Clearly, higher standards for radiation coverage of target tissue with APBI can now be routinely achieved, and previously encountered toxicities can more easily be reduced in many cases they can be completely avoided [7,21-23]. Analyzed issue was recently described in two published papers. Eyre et al. [24] published a study directly comparing four dosimetric techniques for balloon-based partial breast brachytherapy: single source, standard line source, and both forward planned and inverse planned multi-lumen (ML). They presented a standard line source plan to be used in a single catheter or as a starting point for forward planned ML. Twelve treatment plans of patients previously treated with a single lumen were a basis for preparing inverse plans, and used to create a standard line source plan. Authors concluded that multiple sources in a single catheter improved coverage at catheter ends, whereas ML can further improve coverage and reduce dose to organs at risk. Using a standard line source as a starting point for forward planning ML means increase in planning time is kept to a minimum, making it a practicable option for centers without inverse planning software [24]. Arthur et al. [25] recently published a final dosimetric
Table 2. Comparison of $p$ values: real plan with virtual plans

Multi-lumen DP Single-lumen IPSA Single-lumen DP

\begin{tabular}{lccc}
\hline$D_{95}$ & $<0.0001$ & 0.0367 & $<0.0001$ \\
\hline$D_{90}$ & 0.0145 & 0.5440 & 0.0350 \\
\hline$D_{\max }$ skin & 0.0816 & 0.7131 & 0.8763 \\
\hline$D_{\max }$ rib & 0.1452 & 0.0067 & 0.04467 \\
\hline$D_{50}$ & $<0.0001$ & 0.2245 & $<0.0001$ \\
\hline$D_{10}$ & 0.3290 & 0.7787 & 0.0333 \\
\hline$V_{150}$ & $<0.0001$ & 0.2369 & $<0.0001$ \\
\hline$V_{200}$ & 0.0181 & 0.6926 & 0.8001
\end{tabular}

IPSA - Inverse Planning Simulated Annealing, DP - dose points optimization, $D$-dose (dose that covers $\%$ of the target volume), $D_{\text {max }}$ - maximal dose received by skin or rib, $V$-volume $\left(V_{150}\right.$, that is the percentage of the target volume that has received $50 \%$ more than the prescribed dose, $V_{200}-100 \%$ more)

findings of a completed, multi-institutional, phase 4 registry trial using the Contura ${ }^{\circledR}$ Multi-Lumen Balloon (MLB) breast brachytherapy catheter, to deliver APBI in patients with early-stage breast cancer. The idea was to compare 3 dosimetric plans with identical target coverage for each patient to compare: multi-lumen multi-dwell (MLMD); central-lumen multi-dwell (CLMD), and central-lumen single-dwell (CLSD) loading of the Contura ${ }^{\circledR}$ catheter. The use of the Contura ${ }^{\circledR}$ MLB catheter produced statistically significant improvements in dosimetric capabilities between CLSD and CLMD treatments. This device approach demonstrates the ability not only to overcome the barriers of limited skin thickness and close rib proximity, but to consistently achieve a higher standard of dosimetric planning goals [25].

In our observations, IPSA optimized single-lumen applications resulted in acceptable dose coverage $-D_{90}$ and it was comparable with ML IPSA application. Additionally, the skin doses were not differing, only the doses to ribs were higher, however acceptable for vast majority of plans. IPSA algorithm allowed to achieve better plans for single-lumen applicator than for ML DP application. The plans were calculated using dose point optimization with smaller high dose regions, but it was strictly related with underdosing of the treated area. This fact is not clinically crucial as such plans would be optimized manually before treatment of patient. Aim of the study was to test tools implemented to treatment planning system, without physicist intervention. The significant advantage of intracavitary breast balloons is active source dwell positions localized inside the balloon, and separated from the treated tissue. This feature allows to achieve much smaller high dose regions in patient tissue, and decrease the maximum dose. Appropriate construction of the applicator is the reason for the highest dose being absorbed by fluid inside the balloon, therefore the dose distribution is much more homogenous in the target area.

\section{Conclusions}

Dose distribution obtained using multi-lumen IPSA optimized application was characterized with the highest target coverage while preserving acceptable doses to organs at risk. Optimization method was proven to be more im- 
portant than the number of catheters in the applicator, however, overall outcome of multi-lumen application gives more possibilities of dose distribution adjustment.

\section{References}

1. Shah NM, Tenenholz T, Arthur D et al. MammoSite and Interstitial Brachytherapy for Accelerated Partial Breast Irradiation. Factors that Affect Toxicity and Cosmesis. Cancer 2004; 101: 727-734.

2. Veronesi U, Liuini A, Del Vecchio M et al. Radiotherapy after breast-preserving surgery in women with localized cancer of the breast. N Engl J Med 1993; 328: 1587-1591.

3. Gage I, Recht A, Gelman R et al. Long-term outcome following breast conserving surgery and radiation therapy. Int $J$ Radiat Oncol Biol Phys 1995; 33: 245-251.

4. Wazer DE, Schmidt-Ullrich RK, Schmid C et al. Factors determining outcome for breast-conserving irradiation with margin directed dose escalation to the tumor bed. Int J Radiat Oncol Biol Phys 1998; 40: 851-858.

5. Wallace M, Martinez A, Mitchell C et al. Phase I/II study evaluating early tolerance in breast cancer patients undergoing Accelerated Partial Breast Irradiation treated with the Mammosite balloon breast brachytherapy catheter using a 2-day dose schedule. Int J Radiat Oncol Biol Phys 2010; 77: 531-536.

6. Vicini F, Beitsch P, Quiet $C$ et al. Five-year analysis of treatment efficacy and cosmesis by the American Society of Breast Surgeons Mammosite Breast Brachytherapy Registry Trial in patients treated with Accelerated Partial Breast Irradiation. Int J Radiat Oncol Biol Phys 2011; 79: 808-817.

7. Israel PZ, Robbins A, Shroff P et al. Three-year clinical outcome using the Contura multilumen balloon breast brachytherapy catheter to deliver Accelerated Partial Breast Irradiation (APBI): Improving radiation standards for the optimal application of APBI. Brachytherapy 2012; 11: 316-321.

8. Wilder RB, Curcio LD, Khanijou RK et al. A Contura catheter offers dosimetric advantages over a MammoSite catheter that increase the applicability of accelerated partial breast irradiation. Brachytherapy 2009; 8: 373-378.

9. Brown S, McLaughlin M, Pope DK et al. A dosimetric comparison of the Contura multilumen balloon breast brachytherapy catheter vs. the single-lumen MammoSite balloon device in patients treated with Accelerated Partial Breast Irradiation at a single institution. Brachytherapy 2011; 10: 68-73.

10. Tokita KM, Cuttino LW, Vicini FA et al. Optimal application of the Contura multilumen balloon breast brachytherapy catheter vacuum port to deliver accelerated partial breast irradiation. Brachytherapy 2011; 10: 184-189.

11. Cuttino LW, Todor D, Rosu M et al. A Comparison of skin and chest wall dose delivered with multicatheter, Contura multilumen balloon, and MammoSite breast brachytherapy. Int J Radiat Oncol Biol Phys 2011; 79: 34-38.

12. Strauss JB, Dickler A. Accelerated partial breast irradiation utilizing balloon brachytherapy techniques. Review of partial breast brachytherapy. Radiother Oncol 2009; 91: 157-165.

13. Sauer G, Strnad V, Kurzeder C et al. Partial Breast Irradiation after Breast-Conserving Surgery. Strahlenther Onkol 2005; 181: $1-8$

14. Weed DW, Edmundson GK, Vicini FA et al. Accelerated partial breast irradiation: A dosimetric comparison of three different techniques. Brachytherapy 2005; 4: 121-129.

15. Offersen BV, Overgaard M, Kroman N et al. Accelerated partial breast irradiation as part of breast conserving therapy of early breast carcinoma: A systematic review. Radiother Oncol 2009; 90: 1-13.

16. Njeh CF, Saunders MW, Langton CM. Accelerated Partial Breast Irradiation (APBI): A review of available techniques. Radiation Oncol 2010, 5: 90.
17. Polgár C, Van Limbergen E, Pötter R et al. Patient selection for accelerated partial-breast irradiation (APBI) after breast-conserving surgery: Recommendations of the Groupe Européen de Curiethérapie-European Society for Therapeutic Radiology and Oncology (GEC-ESTRO) breast cancer working group based on clinical evidence. Radiother Oncol 2010; 94: 264-273.

18. Kini V. Balloon breast brachytherapy - review of current data. Brachytherapy 2005; 4: 181-182.

19. White J. MammoSite and accelerated partial breast irradiation: Rethinking one-size-fits-all breast irradiation after lumpectomy. Brachytherapy 2005; 4: 183-185.

20. Wazer DE, Kaufman S, Cuttino L et al. Accelerated Partial Breast Irradiation: an analysis of variables associated with late toxicity and long-term cosmetic outcome after High-Dose-Rate interstitial brachytherapy. Int J Radiat Oncol Biol Phys 2006; 64: 489-495.

21. Arthur DW, Vicini FA, Todor DA et al. Improvements in critical dosimetric endpoints using the Contura multilumen balloon breast brachytherapy catheter to deliver Accelerated Partial Breast Irradiation: preliminary dosimetric findings of a phase IV trial. Int J Radiat Oncol Biol Phys 2011; 79: 26-33.

22. Israel $P Z$, Robbins $A B$, Shroff $P$ et al. Initial surgical experience evaluating early tolerance and toxicities in patients undergoing accelerated partial breast irradiation using the Contura multi lumen balloon breast brachytherapy catheter. Am Surg 2009; 75: 1042-1049.

23. Scanderbeg DJ, Yashar C, Rice R et al. Clinical implementation of a new HDR brachytherapy device for Partial Breast Irradiation. Radiother Oncol 2009; 90: 36-42.

24. Eyre K, Whitney D, Mukesh M et al. Optimization and comparison of balloon-based partial breast brachytherapy using a single source, a standard plan line source, and both forward and inverse planned multilumen techniques. Brachytherapy 2013; 12: 107-113.

25. Arthur DW, Vicini FA, Tod DA et al. Contura multi-lumen balloon breast brachytherapy catheter: comparative dosimetric findings of a phase 4 trial. Int J Radiat Oncol Biol Phys 2013; 86: 264-269. 